\title{
Vulnerabilidade das idosas ao HIV/AIDS: despertar das políticas públicas e profissionais de saúde no contexto da atenção integral: revisão de literatura
}

\author{
Vinerability of theedderly toHV ADS pudicpolitissandheatth \\ professionalsinthecontext of integra care aliteraturereview
}

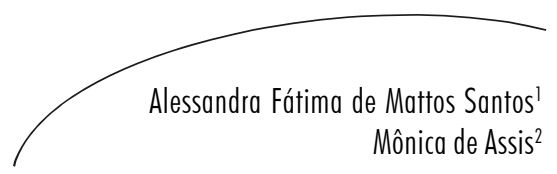

O aumento da incidência de HIV/Aids na população acima dos 50 anos cresce como em nenhuma outra faixa etária, emergindo como desafio para o Brasil no sentido do estabelecimento de políticas públicas e estratégias que garantam o alcance das medidas preventivas e a melhoria da qualidade de vida dessas pessoas. O objetivo deste estudo é abordar os motivos para esse aumento, apontados conforme revisão não sistemática da literatura no período de 1999 a 2009. As buscas ocorreram nas bases de dados LILACS, MEDLINE, SciELO e PubMed, publicações institucionais do Ministério da Saúde, Organização Mundial de Saúde e Organização Panamericana de Saúde. A vulnerabilidade de idosos ao HIV/Aids tem sido relacionada a fatores como invisibilidade do sexo na velhice; desmistificação em curso da sexualidade na terceira idade, associada à ampliação do acesso a medicamentos para distúrbios eréteis e à participação de idosos em grupos de convivência; pequena adesão de homens idosos aos preservativos masculinos; e retardamento de políticas de prevenção direcionadas a este grupo etário. A abordagem integral contribui para o entendimento do processo de adoecimento, especialmente naquele indivíduo com o vírus HIV, que vivencia preconceitos, estigmas e discriminação, desafiando as estratégias de prevenção de novas infecções e ações de assistência à saúde mais apropriadas. É necessário que os profissionais de saúde percebam os idosos como vulneráveis ao risco de infecção pelo vírus HIV e que suas particularidades sejam contempladas nas ações preventivas e assistenciais no contexto da atenção integral à saúde do idoso.

\footnotetext{
Curso de Especialização em Geriatria e Gerontologia. Universidade Aberta da Terceira Idade. Universidade do Estado do Rio de Janeiro. Rio de Janeiro, RJ, Brasil.

2 Instituto Nacional de Câncer, Coordenação de Prevenção e Vigilância. Rio de Janeiro, RJ - Brasil.
}

Palavras-chave: Idosos.

HIV. Aids.

Vulnerabilidade.

Sexualidade. Integralidade. 


\section{Abstract}

The increasing incidence of HIV / Aids in the population over 50 grows as in any other age group, emerging as a challenge to Brazil for the establishment of public policies and strategies that ensure the scope of preventive measures and improving the quality of life of these people. This study aims to understand the reasons for this increase, as indicated by a non-systematic review of the literature from 1999 to 2009. The searches occurred in the databases LILACS, MEDLINE, PubMed and SciELO, institutional publications of the Ministry of Health, World Health Organization and Pan American Health Organization The vulnerability of the elderly to HIV / Aids has been related to factors such as the invisibility of gender in aging; ongoing demystification of sexuality in old age, coupled with increased access to medicines for erectile disorders and the participation of older people living in groups, small adherence of elderly men to male condoms; and delayed prevention policies directed at this age group. The integral approach helps understand the disease process, especially in the individual with HIV, who experiences prejudice, stigma and discrimination, challenging the strategies to prevent new infections and more appropriate healthcare actions. Health professionals should perceive older people as vulnerable to the risk of HIV infection and that their peculiarities are reflected in the preventive and health care actions in the context of integral health care for the elderly.
Key words: Elderly. HIV. Aids. Vulnerability. Sexuality. Integrality.

\section{INTRODUÇÃO}

O envelhecimento é definido como um processo de progressivas modificações biológicas, psicológicas e sociais ao longo da vida do ser humano e, segundo a Organização Mundial de Saúde, é considerado idoso o indivíduo com idade maior ou igual a 60 anos para os países em desenvolvimento e 65 anos para os desenvolvidos. ${ }^{1}$ Até o ano de 2025, conforme dados divulgados pelo Ministério da Saúde, o Brasil será o sexto país do mundo em número de idosos, o que corresponderá a $15 \%$ de sua população. ${ }^{2}$

A descoberta da pandemia do vírus da imunodeficiência adquirida (HIV) no início da década de 1980 causou grande impacto social, por ser construída como doença contraída somente por homens homossexuais, causando estigmas e preconceitos. ${ }^{3}$

A incidência de HIV/Aids na população brasileira acima de 50 anos cresceu de 3,6 para 7,1 em 100.000 habitantes entre 1996 a 2006, representando um aumento de $50 \%$ de casos novos. A doença neste grupo específico apresenta particular relevância epidemiológica pelas altas taxas de incidência, prevalência e letalidade. ${ }^{3-7}$ Dos 47.437 casos de Aids notificados desde o início da epidemia em pessoas acima dos 50 anos, 29.393 (62\%) foram registrados de 2001 a junho de 2008, sugerindo a subnotificação antes do ano 2000. Nesse grupo, $37 \%$ são mulheres e $63 \%$ homens e, atualmente, o índice de HIV entre idosos no Brasil já supera o de adolescentes entre 15 e 19 anos. ${ }^{6-8}$ Dados nacionais referem que o índice de HIV entre idosos já supera o de adolescentes entre $15 \mathrm{e}$ 19 anos. Este aumento do número de casos cresce como em nenhuma outra faixa etária, emergindo como um desafio para o Brasil, exigindo o estabelecimento de políticas públicas e estratégias que possam garantir o alcance das medidas preventivas e a melhoria da qualidade de vida a estas pessoas. ${ }^{9,10}$

O aumento do número de casos também é constatado nos Estados Unidos da América. Até maio de 2007, foi registrado um total aproximado de 78.000 pessoas com mais de 50 anos contaminadas pelo $\mathrm{HIV} / \mathrm{Aids}$, representando 10 a $15 \%$ do total das pessoas infectadas pelo HIV naquele país. Esse aumento se deve à "capa de invisibilidade sobre tal população no que 
concerne a enxergá-los como sujeitos desejantes e sexualmente ativos". ${ }^{1}$ Os dados internacionais sobre DST e HIV/Aids são maiores nas faixas etárias entre 15 e 49 anos, e as informações sobre as taxas de infecção pelo HIV em pessoas acima de 50 anos são limitadas pelo fato de a investigação ser menor pelo profissional de saúde o por supor que a vulnerabilidade seja inexistente. ${ }^{1,11}$

A epidemia de HIV/Aids vem sofrendo diversas modificações em seu perfil ao longo do tempo, dentre as quais os fenômenos de feminilização, heterossexualização, juventudilização, pauperização e envelhecimento. Estas características referenciam que não existem mais indivíduos particularmente vulneráveis ao vírus HIV, já que todas as fases do ciclo de vida estão expostas à contaminação. ${ }^{12} \mathrm{O}$ aumento do número de casos de HIV na população idosa tem sido associado ao envelhecimento da população brasileira, ao aumento da sobrevida das pessoas vivendo com $\mathrm{HIV} / \mathrm{Aids}$ e ao acesso a medicamentos para distúrbios eréteis, fator que tem prolongado a atividade sexual de idosos em associação com a desmistificação do sexo na terceira idade. A abertura para a vivência da sexualidade tem tornado os idosos mais vulneráveis às doenças sexualmente transmissíveis (DST), colaborando para maior incidência desta patologia em indivíduos maiores de 50 anos. ${ }^{5}$

Além dos fatores expostos acima, o diagnóstico de HIV/Aids poderá ser realizado numa fase mais tardia, depois de uma investigação extensa e por exclusão de outras doenças, o que atrasa o diagnóstico e tratamento por mais de dez meses. Isto ocorre porque certos sintomas da infecção, tais como cansaço, perda de peso e distúrbios na memória, não são específicos desta infecção, podendo acontecer em outras doenças que são comuns nesta faixa etária. ${ }^{1,9} \mathrm{Na}$ maioria dos casos, a doença é descoberta quando o paciente é internado para tratar alguma infecção oportunista ainda não diagnosticada ou em exames préoperatórios. ${ }^{1}$

Apesar das mudanças sexuais em curso, a sexualidade está longe de ser vista como saudável e natural em idosos. O preconceito e a falta de informação reforçam a ideia da velhice assexuada, o que aumenta a vulnerabilidade do idoso para as DSTs, entre elas, o HIV/Aids. ${ }^{10}$ As campanhas e ações de prevenção e promoção de saúde que estão sendo realizadas desde o ano de 2008 amenizam a invisibilidade quanto à transmissão do HIV na população geriátrica. ${ }^{13,14}$

\section{METODOLOGIA}

Tendo em vista altas taxas de incidência por HIV/Aids em idosos, tanto no Brasil como em nível mundial, o objetivo deste trabalho é revisar as causas para este aumento apontado na literatura científica contemporânea. $\mathrm{O}$ estudo foi realizado através de revisão não sistemática da literatura no período de 1999 a 2009, foram analisados 31 artigos científicos. Os critérios de inclusão foram as buscas bibliográficas palavras como HIV, Aids, vulnerabilidade; relacionados a idoso ou velhice, buscando-se aspectos causais para o aumento $\mathrm{da}$ incidência pelo vírus $\mathrm{HIV}$, a fim de alertar as políticas públicas e os profissionais de saúde. As buscas ocorreram nas bases de dados da Biblioteca Virtual em Saúde LILACS, MEDLINE, na de textos completos na SciELO e no banco de dados do PubMed, além de sites e publicações institucionais do Ministério da Saúde, Organização Mundial de Saúde e Organização Panamericana de Saúde. Espera-se que este artigo alerte a sociedade, as políticas públicas e os profissionais de saúde quanto à vulnerabilidade do idoso ao HIV/Aids. Sua importância é a conscientização dos profissionais de saúde na abordagem do idoso no contexto da integralidade por também ser vulnerável às DSTs, dentre elas o HIV e a Aids.

\section{RESULTADOS}

Após revisão dos 31 artigos, encontramos cinco itens essenciais para a discussão da vulnerabilidade do HIV/Aids em idosos e, para deixar este estudo didático ao leitor, optamos por organizar a discussão em alguns temas prioritários para o aumento da incidência de idosos com o 
vírus HIV e possíveis ações a serem desenvolvidas para o controle da epidemia nesta faixa etária.

\section{DISCUSSÃO}

O aumento gradativo da incidência de HIV/ Aids em idosos poderia ser explicado, segundo alguns autores, por fatores ligados ao aumento da expectativa de vida ao nascer e da atividade sexual (reposição hormonal para as mulheres e tratamento da impotência sexual para homens) e ao não-reconhecimento do risco pelos idosos e, consequentente, a não-realização do sexo seguro. ${ }^{1,9,13,14}$ Já outros autores atribuem o aumento de casos de Aids em idades mais avançadas àqueles idosos que possuem maiores recursos financeiros para acesso aos prazeres e serviços disponíveis, permitindo vida sexual mais ativa, e à existência da invisibilidade sexual na terceira idade. ${ }^{1,2}$

Os possíveis fatores que têm contribuído para a vulnerabilidade dos idosos frente à epidemia do HIV/Aids são destacados a seguir.

\section{- Invisibilidade da sexualidade na velhice}

As estatísticas atuais mostram o avanço da epidemia da Aids na população com mais de 50 anos, e parece razoável que essas pessoas declarem suas demandas e curiosidades, deixando enfim de serem invisíveis. ${ }^{1,67,12,14,16}$ Pelo fato de a sexualidade em idosos ser pouco investigada, estagniza-se na sociedade e entre os profissionais de saúde, a noção de que sexo e sexualidade não existem na velhice. ${ }^{4,12,14-17}$

As mudanças culturais ocorridas nas últimas décadas e o advento de novas tecnologias para prolongar a vida sexual não impediram o estereótipo do "idoso assexuado", o qual permanece arraigado na sociedade, influenciando não só as representações dos próprios idosos, como também as políticas públicas e programas de investigação. ${ }^{1,10}$. A possibilidade de uma pessoa idosa ser infectada pelo HIV parece invisível aos olhos da sociedade. ${ }^{14,15}$ É constrangedor admitir ou conversar sobre isto, pior do que estigmatizar, discriminar ou lidar com o preconceito em relação à sexualidade e seus riscos após os 50 anos, é tornála invisível. Pode-se sempre lutar contra o estigma, contra o preconceito. É uma não questão, não existe. É silêncio. ${ }^{1,10}$ Para a prevenção do HIV/ Aids em idosos, seria necessária a desconstrução de imagens que foram passadas da doença no início da epidemia como os fatores específicos desta idade, a dificuldade de mudança de hábitos e de incorporação de novas formas de lidar com a sexualidade e os valores culturais de épocas diferentes. ${ }^{1}$

A sexualidade dos idosos, em diversos estudos, sempre foi tratada em módulos "anexos", não sendo considerada parte integrante da vida cotidiana das pessoas mais velhas. Entretanto, uma mudança sociocultural em atitudes relativas à sexualidade e ao envelhecimento vem desafiando o estereótipo tradicional da "velhice assexuada", na medida em que a função sexual passou a ser vista como um componente vital para se alcançar um envelhecimento de sucesso em geral. 4,6,8,14,17-20

O Programa Nacional de Doenças Sexualmente Transmissíveis (DST) e Aids do Ministério da Saúde realizou em 2003 um estudo sobre o comportamento da população de 60 anos ou mais sexualmente ativa. Os dados mostram que $39 \%$ deste grupo têm vida sexual ativa e que predominam as relações heterossexuais com comportamentos de risco, nos quais sexo desprotegido, múltiplos parceiros, abuso de drogas e de medicamentos estão presentes. ${ }^{1}$

Pesquisa do Datafolha realizada no final de 2008 em 24 estados brasileiros revelou que 74\% dos homens idosos entrevistados têm vida sexual ativa, enquanto $76 \%$ das mulheres dizem exatamente o contrário. Além disso, $91 \%$ dos entrevistados revelam que nunca utilizaram medicamentos para estimular a ereção. ${ }^{18}$

O fato de a sexualidade e uso de drogas nesta faixa etária serem tratados como tabus, tanto pelos idosos como pela sociedade em geral, contribui para que a Aids não se configure como ameaça, levando os profissionais de saúde a não solicitarem o teste de HIV nos exames de rotina, em 
decorrência também da associação dos sintomas a outras doenças, o que ocasiona diagnóstico tardio. ${ }^{1,4,15,19}$ Conviver com o vírus HIV na idade avançada traz consigo contradições e desafios a serem enfrentados, tornando visível o invisível, como, por exemplo, a sexualidade ou o uso de drogas na velhice, temas carregados de preconceitos e tabus sociais. ${ }^{4,12,14,15}$

A problemática do envelhecimento e Aids no Brasil relaciona-se também à questão cultural e de exclusão, sobretudo o preconceito social relacionado ao sexo nessa idade. Estudos comportamentais revelam que o desejo sexual permanece nas pessoas mais idosas e que a concepção, arraigada na sociedade, de que sexo é prerrogativa da juventude, contribui para manter fora das prioridades de prevenção das DSTs e Aids os grupos populacionais com idade superior aos 50 anos. ${ }^{9} \mathrm{O}$ aumento da expectativa da população desperta questionamentos sobre o modo como se percebe o processo de envelhecer, buscando trazer transformações nos valores éticos, culturais e estéticos - uma delas é crença de que o avançar da idade e o declinar da atividade sexual estão diretamente ligados, o que é responsável pela desatenção com a sexualidade pelos profissionais de saúde. ${ }^{15}$

Apesar de a Aids nesta faixa etária ter sido identificada pela primeira vez há quase 20 anos, os equívocos continuam e os idosos ainda são vistos como assexuados. E se por acaso eles tiverem relação sexual, são obviamente heterossexuais, certamente monogâmicos e não fazem uso de drogas injetáveis, segundo alguns estudos. Portanto, essas pessoas estão menos informadas sobre o HIV e pouco conscientes de como se protegerem, tornando-se mais vulneráveis à infecção. ${ }^{1,15,17,19}$

\section{- Participação do idoso em grupos da terceira idade}

O aumento da expectativa de vida, aliado ao envelhecimento saudável, tem permitido que os idosos mantenham laços sociais, através da participação ativa das atividades de lazer especialmente destinada para estrato populacional. Tal condição poderá favorecer a inclusão social, afastando a possibilidade de abandono e exclusão, situação frequente entre os idosos que não possuem vínculos sociais. ${ }^{10}$

Os centros de convivência ou grupos de idosos têm como objetivo comum a valorização das pessoas com mais de 50 anos que os frequentam, buscando reverter, em parte, a posição de relegado que o idoso tradicionalmente ocupa no contexto da sociedade contemporânea, que privilegia a juventude e a produtividade., ${ }^{2,8,15,21-23}$

Um estudo revelou que $73,07 \%$ dos idosos participantes de grupos da terceira idade já ouviram falar em DST e HIV/Aids, mas avaliam que não possuem risco de contrair estas doenças. O mesmo trabalho mostrou que somente $21 \%$ usam preservativos, percentual que nos leva a crer que as campanhas de prevenção não estão chegando até este extrato da população. Sabemos que muitos idosos têm vida sexual ativa, alguns com mais de uma parceira; $38,46 \%$ deles nunca usam preservativos em suas relações, mesmo sabendo que é um método de prevenção citado por $73,07 \%$ dos pesquisados..$^{10,13}$

- Medicamentos estimulantes do desempenho sexual em idosos

A vulnerabilidade dos idosos ao vírus HIV/ Aids tem também como fator predisponente o desenvolvimento de drogas de estimulação sexual, garantindo aos idosos um melhor desempenho, sem necessariamente estar associado à prática do sexo seguro. ${ }^{12} \mathrm{~A}$ pesquisa do Datafolha citada anteriormente, realizada no final de $2008 \mathrm{em} 24$ estados brasileiros, revelou que $91 \%$ dos entrevistados nunca utilizaram medicamentos para estimular a ereção. ${ }^{18}$

Os recentes progressos da indústria farmacêutica e da medicina estão permitindo o prolongamento da vida sexual ativa, em associação com a desmistificação do sexo, vulneráveis na terceira idade. ${ }^{11,13,20,24} \mathrm{O}$ Hospital Gaffrée e Guinle, no Rio de Janeiro, começou a monitorar o 
número de casos em HIV/Aids desde $2001 \mathrm{em}$ pacientes acima dos 60 anos e registrou um aumento significativo de soropositividade..$^{25}$

A indústria farmacêutica argumenta que os medicamentos para tratamento da disfunção erétil não estão associados ao aumento do HIV, por estas drogas ajudarem o homem a ter ereção, facilitando assim a utilização dos preservativos. ${ }^{25}$ Outros estudos, por sua vez, demonstram que a descoberta destes medicamentos aumentou a qualidade e o número das relações sexuais entre adultos maiores de 50 anos sem se fazer acompanhar de maior adesão ao uso de preservativos. ${ }^{8,13}$

\section{- Uso do preservativo masculino em idosos}

Há muitos obstáculos para o uso da camisinha: os homens temem perder a ereção e ainda acham que o cuidado só é necessário nas relações com as profissionais do sexo. Já as mulheres não sentem necessidade de exigir o preservativo, porque já perderam a capacidade de engravidar e consideram que não necessitam mais de prevenção. Sexo sem camisinha é particularmente arriscado depois da menopausa, devido ao ressecamento das paredes vaginais, as queixas se tornam mais frequentes e isto poderá favorecer ao surgimento de feridas que abrem caminho para o vírus HIV. . $^{8,20,26}$

Deve-se levar em consideração que a fidelidade muitas vezes é provada no tocante ao uso do preservativo, o qual historicamente esteve atrelado às DSTs e à desconfiança em relação psicoafetiva. Deste modo, dificulta o diálogo acerca da necessidade do uso do preservativo, como se este só devesse ser usado apenas por quem se desconhece e desconfia. ${ }^{20} \mathrm{~A}$ população idosa iniciou a vida sexual antes do surgimento do $\mathrm{HIV/} \mathrm{Aids,} \mathrm{não} \mathrm{reconhecendo} \mathrm{o} \mathrm{risco} \mathrm{de} \mathrm{contrair}$ a infecção e não estar familiarizada com o uso do preservativo. $^{5}$

Um estudo publicado na New England Journal of Medicine com 3.005 homens e mulheres entre 57 a 85 anos revelou que $81 \%$ dos homens e $51 \%$ das mulheres não dispensam sexo. ${ }^{22}$
Dados do Ministério da Saúde (2005) indicam que somente $37,5 \%$ dos indivíduos sexualmente ativos com mais de 50 anos faziam ou fazem uso regular do preservativo com eventuais parceiros, sendo um dos objetivos para a prevenção da Aids e outras DSTs o aumento desta adesão. ${ }^{5} \mathrm{E}$, segundo A raújo et al. (2007), a maioria dos idosos realiza o ato sexual sem a proteção do preservativo, talvez até mesmo por não acreditar que possam contrair o HIV e serem contaminados. ${ }^{19}$

Não causa surpresa o fato de os idosos não adotarem medidas de prevenção para o HIV, pois, além da questão cultural, os programas de prevenção são mais voltados para a camada mais jovem da população. ${ }^{9}$

Estudo realizado pela Universidade do Sul de Santa Catarina, no qual foram entrevistados 22 pacientes soropositivos com idade acima dos 50 anos, revelou que $72,8 \%$ dos entrevistados contraíram o vírus através de relação sexual e $27,2 \%$ através do uso de drogas injetáveis. No mesmo estudo, 52,4\% referiram manter relação sexual após a descoberta da soropositividade e começaram a utilizar o preservativo após a infecção, enquanto $42,9 \%$ relataram manter relação sexual, mas nunca utilizar o preservativo, mesmo após a infecção; os demais negam manter atualmente relações sexuais. Nenhum participante utilizou o preservativo antes da infecção pelo HIV..$^{13}$ Este dado tem relevância, pois mesmo sabendo que são soropositivos e que podem transmitir o vírus para seu parceiro, ainda assim praticam sexo desprotegidos - consequentemente, estão disseminando o vírus e aumentando sua transmissão nesta faixa etária.

Algumas questões culturais ainda permanecem sobre o uso do preservativo, como a infidelidade e a multiplicidade de parceiras aceitas socialmente na trajetória de vida dos homens que hoje têm mais de 60 anos. Estes não praticam sexo seguro porque isso nunca fez parte da vida deles, assim eles expõem suas esposas ao risco de infecção pelo HIV/Aids. ${ }^{20}$

Dados parciais de pesquisa de comportamento sexual realizada pelo Ministério da Saúde em 2008 
apontam que $72 \%$ das brasileiras entrevistadas acima dos 50 anos de idade não usam camisinha com parceiros casuais. ${ }^{27} \mathrm{O}$ tema do carnaval do ano de 2009 dá continuidade à campanha do Dia Mundial de Luta contra a Aids 2008, lançada no $1^{\circ}$ de dezembro, que teve como foco os homens com mais de 50 anos. Entre eles, o uso de camisinha também é baixo. Na pesquisa realizada em 2008, 63\% afirmam não ter o costume de utilizar camisinha nas relações eventuais. ${ }^{27}$

A repugnação quanto ao uso do preservativo é apontada em vários trabalhos e precisa ser mais bem compreendida, a partir de discussões entre os envolvidos sobre os vários aspectos e níveis das dificuldades encontradas. ${ }^{1}$

\section{- Políticas de prevenção de HIV/Aids em idosos na população geriátrica}

Nossa sociedade dá muito valor ao corpo, e a sexualidade passa a ser cada vez mais vendida pela mídia sob forma de medicamentos, roupa, massagens, terapias miraculosas, entre outros produtos. Nesse contexto, o uso de terapias hormonais e a descoberta de medicamentos para fortalecer a potência sexual podem estar promovendo uma melhor qualidade de vida sexual para este segmento. Em contrapartida, não há uma efetiva implantação de políticas que deem respaldo a esta população, tanto no que se refere à prevenção da Aids quanto ao processo de envelhecimento. ${ }^{1,3,10,19}$

Desde que foram lançados os medicamentos para prolongar a vida sexual e as novas biotecnologias de reposição hormonal, por exemplo, os potenciais usuários, os idosos, não foram alertados sobre a consequente vulnerabilidade em termos de exposição às DSTs, que exige o uso do preservativo. ${ }^{1}$

Segundo a UNAids (2005), a ampliação da Aids entre os idosos pode estar associada a uma falha nos esforços de prevenção com este grupo de idade. Campanhas para a população idosa são fundamentais, mas somente o conhecimento não é suficiente para mudar o comportamento para que o indivíduo seja capaz de adotar práticas seguras, a fim de evitar a infecção. É necessário, sobretudo, enfocar aspectos socioculturais para reduzir riscos e vulnerabilidades. ${ }^{2}$

O Programa Nacional de DST e Aids realizou uma campanha, lançada no dia $1^{\circ}$ de dezembro de 2008, como resposta ao aumento da incidência de HIV em idosos, cujo objetivo é despertar nos adultos maduros e idosos a importância do uso do preservativo nas relações sexuais. ${ }^{27}$ A OMS reconhece que o Brasil é um dos primeiros países a iniciar tais políticas, devido o aumento da incidência de Aids. ${ }^{24}$

Outra campanha foi lançada no dia 13 de fevereiro de 2009, pelo Ministério da Saúde, para alertar aos idosos sobre os riscos de Aids e outras DSTs no Carnaval 2009, com o slogan "Sexo não tem idade para acabar. Proteção também não”. O Ministério da Saúde sustenta que é preciso conscientizar as mulheres sobre comportamentos de risco para o vírus $\mathrm{HIV} / \mathrm{Aids}$, devido ao aumento da incidência de Aids nesse segmento. ${ }^{27}$

A literatura enfatiza o conhecimento sobre $\mathrm{HIV} /$ Aids em indivíduos jovens e profissionais da saúde, mas há poucas informações relacionadas à Aids em idosos. A partir desta carência, tornase necessário desenvolver estudos nessa área, pois o conhecimento é importante tanto para a diminuição do preconceito com portadores do HIV quanto para medidas de prevenção.

\section{ABORDAGEM INTEGRAL AO IDOSO COM HIV/AIDS}

$\mathrm{Na} 4^{\text {a }}$ Conferência sobre Patogênese, Tratamento e Prevenção da Sociedade Internacional de Aids, foi constatado que a ampla disponibilidade dos antirretrovirais levou ao crescimento $\mathrm{da}$ prevalência do HIV entre grupos de idosos e que muitos dos "gigantes geriátricos", como as doenças cardiovasculares, câncer e demência, fazem parte do diagnóstico diferencial do vírus HIV/Aids, que no entanto não é realizado pela grande maioria dos profissionais da saúde. ${ }^{28}$

Um levantamento da Secretaria Municipal de Saúde do Rio de Janeiro (1984-2004) demonstrou 
aumento progressivo dos registros de Aids nas faixas etárias acima dos 50 anos e referiu a necessidade de alertar profissionais de saúde que costumam associar queixas e sintomas ao processo natural de envelhecimento ou outras doenças comuns à velhice. Esta postura, aliada à crença de que só os jovens podem se contaminar com o vírus HIV, dá margem a diagnósticos tardios e deixa essa população desassistida. ${ }^{1}$

A OMS publicou um artigo, em março de 2009, sobre a inexplorada história do HIV em idosos, relatando que faltam estimativas para avaliar o real número de casos de HIV em idosos. Embora a atividade sexual seja o mais provável modo de transmissão, faltam investigações para determinar a contribuição relativa dos diferentes fatores de risco e modos de transmissão. O rastreio pelos médicos não é comum, por acharem que não estão em risco. ${ }^{24,29,30}$

Se o profissional de saúde considerar com naturalidade a ocorrência de atividade sexual na terceira idade, o encaminhamento dessa pessoa para o exame do HIV se tornaria um procedimento rotineiro, da mesma forma como tem sido realizado junto ao segmento mais jovem da população. ${ }^{31}$

O aconselhamento e o reconhecimento da vulnerabilidade se fundamentam na interação e na relação de confiança que se estabelece entre o profissional e o paciente. Para que a vulnerabilidade seja concretizada, o profissional abordará o paciente no contexto da integralidade, escutando suas preocupações e dúvidas, desenvolvendo habilidades para perguntar sobre a vida íntima, com a finalidade de propor questões que facilitem a reflexão e a superação de dificuldades, adotando práticas seguras em busca da promoção da qualidade de vida. Para que todos esses objetivos sejam alcançados, é fundamental que, durante todo o atendimento, a linguagem utilizada seja acessível ao usuário. ${ }^{31}$

Para que os profissionais de saúde vejam seus idosos como vulneráveis ao vírus HIV, é necessário o enfoque da integralidade na atenção à saúde. Esta é uma das ferramentas utilizadas pela Estratégia de Saúde da Família (ESF) e um dos princípios do Sistema Único de Saúde (SUS) para as práticas de saúde.

Partindo do conceito da universalidade no paciente vivendo com HIV/Aids, a atuação na abordagem integral não é somente no aspecto da prevenção; também a responsabilização, o vínculo, o acolhimento e a humanização são elementos essenciais para melhoria na qualidade de vida e adesão ao tratamento antirretroviral. ${ }^{23}$

A abordagem integral contribui para o entendimento do processo de adoecimento, especialmente no indivíduo no qual se identifica o vírus HIV, já que esta doença, embora com suas taxas de prevalência, incidência e mortalidade distribuídas em altos índices, promove preconceitos, estigmas e discriminação, invadindo a privacidade e a vida dos indivíduos, que por fim são causadores do grande impacto da epidemia, desafiando as estratégias de prevenção de novas infecções e as ações de assistência à saúde mais apropriada. Em função de o estigma envolver a doença, no nível individual, as pessoas não conversam sobre o vírus HIV e se expõem cada vez mais aos riscos, evitando a realização de testes diagnósticos e comprometendo as medidas de prevenção e cuidado. ${ }^{16,29,30}$

A relação médico-paciente ou de outro profissional da saúde envolvido no cuidado é a chave desta prática integral, pois as informações que emergem desta abordagem permitem ao paciente revelar motivações íntimas, como angústias, temores, preocupações - enfim, revelações que só podem ser ditas em relações de extrema confiança. ${ }^{29}$

Desde o seu surgimento, a Aids vem assumindo um paradigma de doença orgânica que requer uma abordagem biopsicossocial, determinando uma crise multidimensional que afeta não apenas as pessoas contaminadas, mas também seus parceiros sexuais, familiares, cuidadores, profissionais de saúde e comunidades. ${ }^{17,12}$

\section{CONSIDERAÇÕES FINAIS}

Podemos, com esta revisão literária, entender a necessidade de os profissionais de saúde 
enxergarem seus pacientes idosos como propícios ao risco de infecção pelo vírus HIV e que estes sejam sempre visíveis perante a sociedade e políticas públicas.

Como a expectativa e qualidade de vida tendem a aumentar, torna-se necessário implementar estratégias para diminuir o estigma em relação à vida sexual das pessoas mais velhas, práticas educativas para esta população, bem como

\section{REFERÊNCIAS}

1. Zornita M. Os novos idosos com AIDS: sexualidade e desigualdade à luz da bioética. Rio de Janeiro. Dissertação [ Mestrado em saúde pública] -Fundação Oswaldo Cruz; 2008.

2. Saldanha AAW, Araújo LF. Viver com AIDS na Terceira Idade. In: Congresso Virtual. Anais do 7. Congresso Virtual HIV/AIDES 2006. [acesso em 25 fev 2009]. Disponível em: http:// www.aidscongress.net/ article.php?id_comunicacao $=296$.

3. Zildene S, Leite JL. Aids e envelhecimento. Reflexões sobre a infecção pelo HIV em indivíduos acima de 60 anos 2008 [acesso em Jan 092009 ] Disponível em: http:// www.saude.rio.rj.gov.br/media/ AIDSENVELHECIMENTO.doc.

4. Saldanha AAW, Araújo FL. A Aids na terceira idade na perspectiva dos Idosos, Cuidadores e profissionais de saúde. In: Congresso Virtual: anais do 7. Congresso Virtual HIV/AIDES; 2006 [acesso em $10 \mathrm{Fev}$ 2009]. Disponível em URL: http://www.aidscongress.net/ article.php?id_comunicacao $=294$.

5. Saber viver. Aumenta o numero de casos de idosos com HIV/AIDS. [acesso em 15 mar 2009 ]. Disponível em: http://www.saberviver.org.br/ index.php?g_edicao=comportamento_43.

6. Programa Nacional DST/AIDS. Incidência entre os maiores de 50 anos preocupa 2008 [acesso em 2009]. Disponível em http:// www.aids.gov.br/data/Pages/ lumis67f61bd3itemid1bfb1fb10itemidtemidE.htm.

7. Abe E. Incidência de Aids dobra entre os maiores de 50 anos. [acesso em 15 jan 2009]. Disponível em: http://g1.globo.com/Noticias/ Brasil/0,Mul87491MUL874919-5598,00incidencia + de + aids + dobra + entre + os + maiores $+\mathrm{de}+\mathrm{anos}+\mathrm{diz}+$ ministerio.html. incentivar pesquisas que focalizem a relação entre idosos e HIV.

Após os artigos revisados, entendemos que as práticas de cuidados de si e dos outros podem ser construídas nas relações sociais, afetivas, de aprendizagem e poder que se estabelecem entre as pessoas, instituições, serviços de saúde e assistência, tornando-se necessário que não sejamos conviventes com essa invisibilidade que silencia e gera vazio.
8. Matsuoka PK, Locali RF, Girão MJBC. O Conhecimento dos idosos sobre prevenção de doença sexualmente transmissível: elaboração de um questionário. Trabalho apresentado na 60. Reunião Anual da SBPC; 2008 [acesso em 20 Dez 2008 ]. Disponível em: http:// br.geocities.com/xvi.comau/anais/trabalhos/ matsuoka.pdf.

9. Pottes FA, Brito AM, Gouveia GC, Araújo EC, Carneiro RM. Aids e envelhecimento: característica dos casos com idade igual ou maior que 50 anos em Pernambuco, de 1999 a 2000. Rev Bras epidem 2007 set; 10(3): 338-51.

10. Leitea MT, Moura C, Berlize EM. Doenças Sexualmente Transmissíveis e HIV/AIDS na opinião de idosos que participam de grupos de terceira idade. Rev Brás Geriatr Gerontol 2007 [Acesso 09 Maio]. Disponível em: http:// www.unati.uerj.br/tse/ scielo.php? script $=$ sci arttext\&pid $=$ S180998232007000300007\&lng $=$ pt\&nrm $=$ isso.

11. Lazzarotto AR, Kramer AS, Hãdrich M, Tonin M, Caputo P, Srinz E. O Conhecimento de HIV/ AIDS na Terceira idade: estudo epidemiológico no Vale dos Sinos. Cien saúde Coletiva 2008;13(6):1833-40.

12. Diniz RF, Saldanha AAW. Representações sobre AIDS na Velhice por Agentes Comunitários de Saúde. In: Congresso Virtual. Anais do 8. Congresso Virtual HIV/AIDES;2008 [acesso em10 jan 2009 ]. Disponível em: http:// www.aidscongress.net/ article.php?id_comunicacao $=328$.

13. Bertoncini BZ, Moraes KS, Kulkamp IC. Comportamento sexual em adultos maiores de 50 anos infectados pelo HIV. J Bras Doenças Sex Trans. 2007;19(2):75-9.

14. Lisboa MÊS. A invisibilidade da população acima de 50 anos no contexto da epidemia HIV/ 
AIDS.[internet]. In: Congresso Virtual: Anais do 7. Congresso Virtual HIV/AIDES 2006. [acesso em $20 \mathrm{Fev}$ ]. Disponível em: http:// www.aidscongress.net/pdf/281.pdf.

15. Figueiredo MAC, Provinciali RM. HIV/AIDS em pessoas idosas: vulnerabilidade, Convívio e Enfrentamento. In: Congresso Virtual: Anais do 7. Congresso Virtual HIV/AIDES 2006 [acesso em 11 Jan 2009 ]; São Paulo ;Portugal.Disponível em: http:// www.aidscongress.net/ article.php?id_comunicacao $=280$.

16. Lima DB. A Assistência para além da distribuição de medicamentos: $\mathrm{O}$ desafio do envolvimento. In: Raxach JC, Lima DB, Guimarães M, Parker R, Pimenta C, Terto Junior V. Reflexões sobre assistência a AIDS: relação médico-paciente interdisciplinaridade integralidade. [internet]. Rio de Janeiro: ABIA 2003 [Acesso em 09 Mar 2009 ]. Disponível em http:// www.abiaids.org.br/_img/media/ anais\%20assistencia $0 \overline{2}$.pdf.

17. Gasparini SM, Perez BFA. A vivência do idoso no processo de envelhecer e o HIV/AIDS: uma reconstrução dupla com suas possibilidades e limites. J Bras AIDS 2004 set/out; 5(5): 203-206.

18. Sampaio P. Sexualidade na Terceira Idade. Folha de São Paulo. 2009 Mar 15: Caderno Maior Idade: 2.

19. Araújo VLB, Brito DMS, Gimeniz MT, Queiroz Ta, Tavares CM. Características da AIDS na terceira idade em um hospital de referência do Estado do Ceará. Rev Bras epidemiol 2007 dez; 10(4): 544-54.

20. Fontes KS, Saldanha AAW, Araújo LF. Representações do HIV na terceira idade e vulnerabilidade no idoso. In: Congresso Virtual: anais do 7. Congresso Virtual HIV/ AIDES; 2006 [acesso em 10 jan 2009]; Portugal. Disponível em: http://www.aidscongress.net/ article.php?id_comunicacao $=307$.

21. Barreira M. Especialista alerta para a importância de exames de sorologia do HIV em idososão. Agência de Notícias da AIDS 2007 [acesso em 5 Fev 2005]. Disponível em: http:// www.agenciaaids.com.br/noticiasresultado.asp? Codigo $=8007$.

22. New York Times. EUA: Pesquisa indica que maioria dos idosos faz sexo. Agência de Notícias da AIDS 2007 [acesso em $02 \mathrm{Mar}$ 2009]. Disponível em: http:// www.agenciaaids.com.br/noticiasresultado.asp? Codigo $=8191$.
23. Oliveira DC, Leal LM, Fernandes RF, Fonseca DS, Torres LA. DST/AIDS na mira da prevenção: uma perspectiva de integralidade. Trabalho apresentado no VI Seminário do Projeto Integralidade: saberes e práticas no cotidiano das instituições de saúde; 2006 [acesso em 05 jan 2009] Disponível em: http:// www.lappis.org.br/download/ Resumos_VIS_lercap6.pdf.

24. Schmid GP, Williams BG, Calleja JMG, Miller C, Segar E, Southworth M et al. The unexplored story of HIV and ageing. Bulletin of the World Health Organization 2009 [Acesso 12 Maio 2009 ] 87(3): 161-244. Disponível em: http:// www.who.int/bulletin/volumes/87/3/09064030/en/index.html.

25. Jurberg C. Unprotected sex has no age. Bulletin of the World Health Ogranization 2009 [Acesso12 Maio ]. Disponível em: http:// www.who.int/bulletin/volumes/87/3/09010309/en/index.html.

26. Xará S, Dias I, Mota M. Nutrição e VIH: Particularidades no Idoso. In: Congresso Virtual: Anais do 7. Congresso Virtual HIV/ AIDES; 2006; São Paulo. Disponível em: http:// www.aidscongress.net/ article.php?id_comunicacao $=298$.

27. Masgrau R. Campanha de Prevenção à Aids foca mulheres acima de 50 anos. JusBrasil Política 2009. [acesso em 11 Jul 2009]. Disponível em: http://www.jusbrasil.com.br/ politica/1611449/campanha-de-prevencao-aaids-foca-mulheres-acima-de-50-anos.

28. Barreira M. A Imunodeficiência causada pelo HIV, avanços do tratamento e prevalência do vírus entre idosos foram tema da primeira plenária realizada na IAS 2007. Agência de Notícias da Aids 2005 [ Acesso em 12 Mai 2009]. Disponível em: http:// www.agenciaaids.com.br/noticiasresultado.asp? Codigo $=7989$.

29. Veloso V. Avanços e dificuldades de uma questão de mobilização social, profissional e governamental . In: Guimarães JCR, Parker MR, Lima DB, Pimenta C, Terto Jr V, orgs. Reflexões sobre assistência à AIDS: relação médicopaciente, interdisciplinaridade e integralidade. Rio de Janeiro: ABIA; 2003 [Acesso11 Maio 2003 ]. Disponível em: http://www.abiaids.org.br/ _img/media/anais\%20assistencia01.pdf.

30. Abdalla FTM, Nichiata LYI. A abertura da privacidade e o sigilo das informações sobre o HIV/AIDS das Mulheres atendidas pelo Programa de Saúde da Família no Município de São Paulo. Saúde Soc 2008; 17(2): 140-52. 
31. Ministério da Saúde (Brasil), Secretaria de Atenção à Saúde, Departamento de Atenção Básica. Envelhecimento e Saúde da Pessoa Idosa
2006. [Acesso em 10 Maio 2009 ]. Disponível em: http://dtr2004.saude.gov.br/dab/docs/ publicacoes/cadernos_ab/abcad19.pdf.

Recebido: 21/8/2009

Revisado : 26/7//2010

Aprovado : 13/9/2010 
